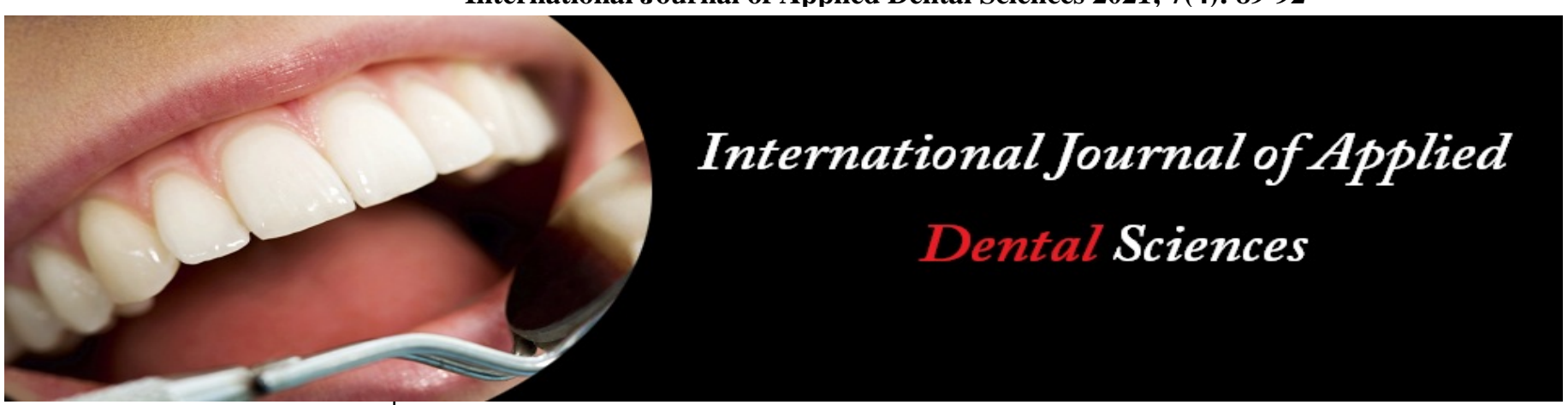

ISSN Print: 2394-7489

ISSN Online: 2394-7497

IJADS 2021; 7(4): 89-92

(C) 2021 IJADS

www.oraljournal.com

Received: 11-08-2021

Accepted: 15-09-2021

Dr. Maniyammai A.S.

Post Graduate Student,

Department of Periodontology,

K.S.R. Institute of Dental

Science and Research,

Tiruchengode, Tamil Nadu,

India

Dr. Renukadevi R

Professor, Department of

Periodontology, K.S.R. Institute

of Dental Science and Research,

Tiruchengode, Tamil Nadu,

India

Dr. Esther Nalini H

Professor/HOD, Department of

Periodontology, K.S.R. Institute of Dental Science and Research,

Tiruchengode, Tamil Nadu,

India

Dr. Arun Kumar Prasad

Professor, Department of

Periodontology, K.S.R. Institute

of Dental Science and Research,

Tiruchengode, Tamil Nadu,

India

Dr. Amudha M

Post Graduate Student,

Department of Periodontology,

K.S.R. Institute of Dental

Science and Research,

Tiruchengode, Tamil Nadu,

India

\section{Adaptive immune response: Molecular aspects of periodontal disease}

\author{
Dr. Maniyammai A.S., Dr. Renukadevi R, Dr. Esther Nalini H, Dr. Arun \\ Kumar Prasad and Dr. Amudha M
}

DOI: $\underline{\text { https://doi.org/10.22271/oral.2021.v7.i4b.1356 }}$

\section{Abstract}

Periodontium that anchors the teeth to the jaws comprises of gingiva, periodontal ligament, cementum, and alveolar bone. At clinically healthy sites, the balance between the microbiota and periodontium is beneficial, resulting in resistance to colonization by commensal periopathogens and also triggering welldefined responses of the host innate immune system. By contrast, at diseased sites there is an alteration of defense mechanisms in the periodontium resulting from heterogeneous etiologies including complex biofilm in the sub gingival microenvironment, which is influenced and modulated by the host's immune and inflammatory responses. The present review will focus on the cells of adaptive immune response like $\mathrm{B}$ - and T-cells in periodontal disease progression.

Keywords: Adaptive immunity, Antigen presenting cells, T-cells, B-cells

\section{Introduction}

Periodontitis is defined as an infectious disease leading to slow to moderate progressive loss of attachment and bone ${ }^{[1]}$. The accumulation of bacterial plaque in the periodontal environment is rapidly monitored by the innate immune system, the first line of defense against invading pathogens ${ }^{[2]}$. The innate immune system includes anatomical barriers, secretory molecules, and cellular elements such as neutrophils, macrophages, and dendritic cells (DCs) ${ }^{[3]}$. These cells are equipped with receptors enabling them to identify various oral invaders via recognition of pathogen-associated molecular patterns like lipopolysaccharide (LPS) or flagellin. The activity of innate immune cells is limited in its specificity to the pathogen, the development of a highly specific adaptive immunity takes place in parallel. During the early stage, there will be enhanced T-cell activity, whereas in the established lesions, B-cells become the most common inflammatory cells ${ }^{[4]}$. These changes signify a local alteration of immunoregulatory events in the host. The significance of acquired immune response relies not only in fighting the virulent periodontal pathogens but also in protecting host from further tissue destruction

\section{Aaptive immunity}

Adaptive immunity responds to the challenge with a high degree of specificity ${ }^{[4]}$.

The major functions include:

- Recognition of specific "non-self" antigens in the presence of "self", during the process of antigen presentation.

- Generation of responses that are mediated to eliminate specific pathogens or pathogeninfected cells.

- Development of immunological memory, in which pathogens are "remembered" through memory B cells and memory T cells.

- Antibody production is the critical part of Adaptive immunity which in turn provide longlasting protection, sometimes for the person's entire lifetime.

Antigen Presenting Cells in adaptive immune response

Antigen presentation allows for specificity of adaptive immunity which contribute to immune responses against both intracellular and extracellular pathogens.
Dr. Maniyammai A.S. Post Graduate Student, Department of Periodontology, K.S.R. Institute of Dental Science and Research, Tiruchengode, Tamil Nadu, India 
Macrophages, Dendritic cells and B cells have the ability to present antigens specifically for the purpose of activating $\mathrm{T}$ cells. These types of cells are referred to as antigen-presenting cells (APCs) ${ }^{[5]}$.

\section{Mechanisms of Antigen Processing and Presentation}

APCs can digest proteins they encounter and display peptide fragments from them on their surfaces for specific immune cell to recognize. This process of antigen presentation allows T cells to "see" what proteins are present in the body and to form an adaptive immune response against them. The foreign protein, antigens are degraded into small peptides and forms complex with the Major histocompatibility complex then present on the surface of APCs ${ }^{[6]}$.

The processing involves six steps as

1) Acquisition of antigenic peptides;

2) Tagging of the antigenic peptide for destruction by ubiquitylation;

3) Proteolysis;

4) Delivery of peptides to the endoplasmic reticulum (ER);

5) Binding of peptides to MHC class II molecules; and

6) Display of peptide-MHC class II complexes on the cell surface. Peptide-loaded MHC class II molecules are then transported to the cell surface where they engage antigenspecific CD4+ T cells. (Figure 1)

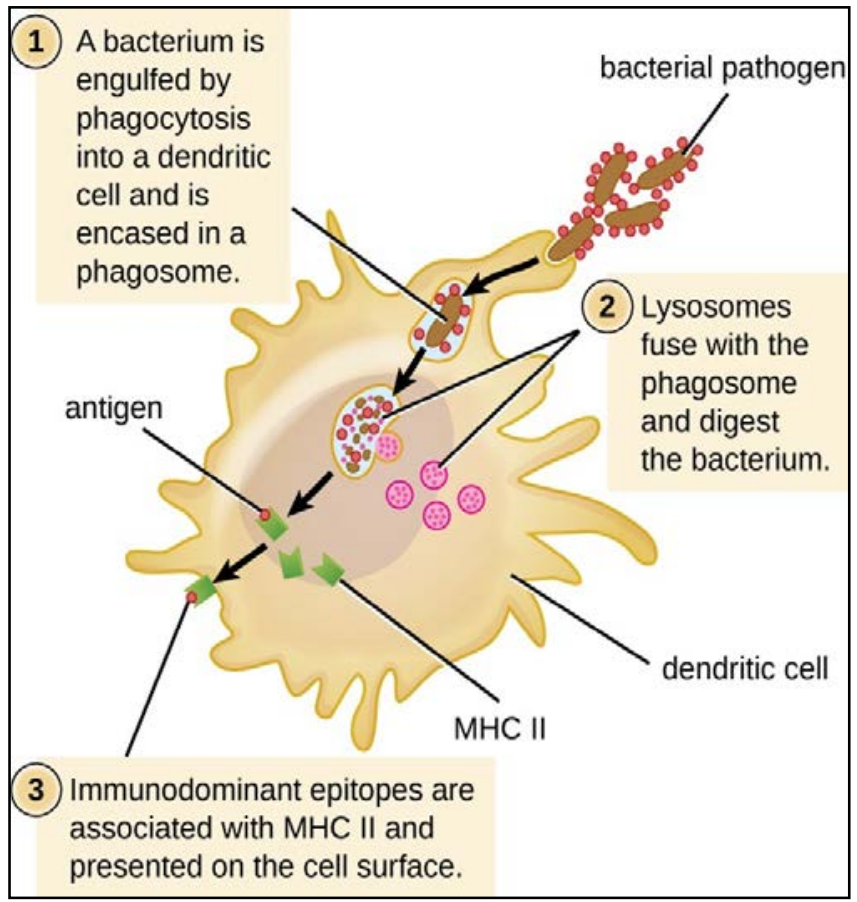

Fig 1: Antigen uptake 2. Processing 3. Presentation

\section{T-cell response:}

$\mathrm{T}$ cell activation and differentiation requires three signals

i). Antigen recognition

ii). Co-stimulation of T-cell

iii). Differentiation of T-cell

First signal- Antigen recognition

The naive $\mathrm{T}$ lymphocytes within the secondary lymphoids essentially express L-selectin, which helps in binding to the specialized cells that display addressins PNAd (peripheral node addressin) or MAdCAM-1 (mucosal addressin cell adhesion molecule-1). T lymphocytes develop contact with numerous DCs and binds to only those expressing a compatible antigen specific to their TCR. The duration of interaction might be transient (3-11 minutes) or steady (several hours) which is dependent on the affinity for that particular antigen ${ }^{[6,7]}$. (Figure 2)

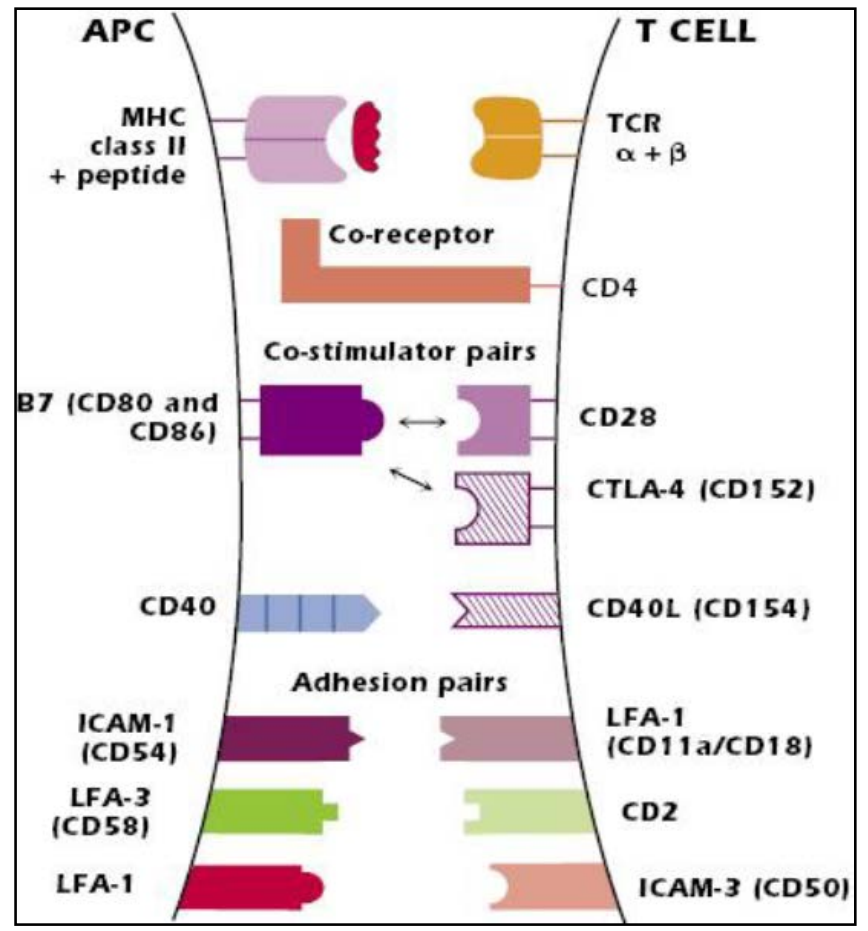

Fig 2: Signaling events for activation of T-cells

\section{Second signal-Co-stimulation}

Certain molecules expressed on the surface of specialized antigen-presenting cells (APCs) interact with molecules expressed on the T-cell surface resulting in formation of costimulatory pairs.

The signaling molecule B7, expressed on professional APCs (dendritic cells, macrophages, and activated B cells) interact with CD28, expressed on T cells.

Once the interaction occurs, TCR upregulates expression of CD40L, which in turn induces increased expression of CD40 on the APC. This CD40-CD40L interaction further increases B7 expression on the APC, enhancing the B7-CD28 interaction between APC and T cell. Without co-stimulation, the process of $\mathrm{T}$ lymphocyte activation is not complete, and becomes anergic.

Three main functions of co-stimulation:

- It makes the T-cell resistant to apoptosis

- It upregulates growth factor receptors on the T-cell thereby stimulating its proliferation

- It decreases the amount of time needed to trigger the Tcell.

Both the first and second signals send messages to the T-cell nucleus (i.e., trigger signal transduction) that change the pattern of the genes expressed by that $\mathrm{T}$ cell. Both signals are needed to activate naive $\mathrm{T}$ cells; ultimately, they lead to survival, proliferation, and differentiation of the $\mathrm{T}$ cell.

\section{Molecular Events in CD4+ T-Cell Activation SS}

After antigen recognition, various kinases, such as lymphocyte-specific protein tyrosine kinase (LCK), CSK, and ZAP-70, and phosphatases, such as SHP-1, SHP2, and CD45, influence the phosphorylation of state of the CD3 immunoreceptor tyrosine-based activation motifs (ITAMs) on the TCR. The activation of Lck leads to the phosphorylation of tyrosines in the cytoplasmic domains of CD3 and TCR z 
chains. Phosphorylated TCR $\mathrm{z}$ chains recruit the tyrosine kinase ZAP-70, which subsequently phosphorylates the transmembrane adaptor LAT. This leads to the assembly of a multiprotein complex that is essential for the broadcasting several pathways, eventually resulting in the activation of transcription factors NFAT, AP-1 and NF- $\kappa$ B.

These activated factors NF- $\kappa \mathrm{B}$, NFAT, and AP-1 enter the nucleus of activated $\mathrm{T}$ cell for up regulation of several different genes. (Figure 3). Two important genes that are transcribed and translated in the activated $\mathrm{T}$ cell (within 24 hours of the onset of activation) are the genes for the cytokine IL-2 and for one of the chains of the IL-2 receptor: IL-2R $\alpha$ (CD25). IL-2 is also known as T-cell growth factor. IL-2 acts on both the $\mathrm{T}$ cell that synthesized and secreted it as well as daughter cells produced by proliferation. As a result, the original clone of $\mathrm{T}$ cells undergoes enormous and rapid expansion, increasing the clone size several thousand-fold within a week ${ }^{[7,8,9]}$.

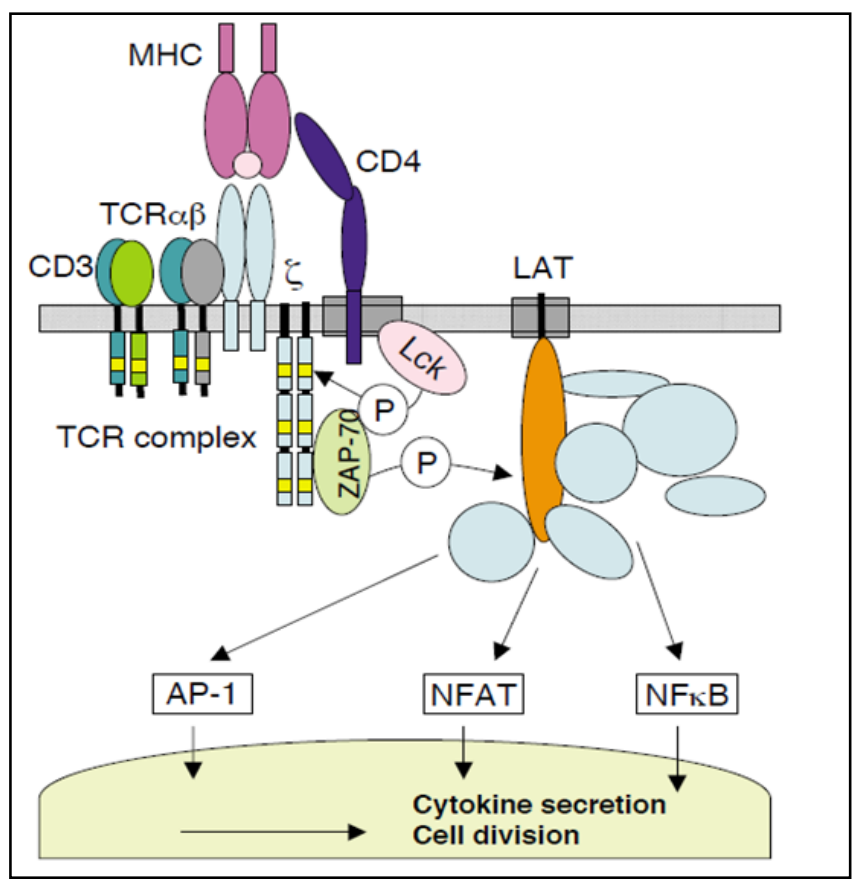

Fig 3: Receptor mediated events leading to T-cell activation

Third signal is generated by inflammatory cytokines produced by the APC or other cells at the site of $\mathrm{T}$ cell activation. These cytokines direct differentiation of naïve CD4+ T cells into a particular effector subset. Effector CD4+ $\mathrm{T}$ cells can be categorized into subsets based on the type of cytokine they produce and the major transcription factor (TF) they express.

\section{Clonal expansion}

Naive CD4+ $\mathrm{T}$ cells are activated after interaction with antigen-MHC complex and differentiate into specific subtypes depending on the cytokine microenvironment. After such interactions, they become activated and differentiated into effector $\mathrm{T}$ cells, which are responsible for the production of effector molecules, such as pro-/anti-inflammatory cytokines and cytotoxic molecules. A cardinal feature of $\mathrm{T}$ cell immunity is the ability of naïve $\mathrm{T}$ cells to undergo a program of proliferation and functional differentiation upon activation, resulting in a large pool of cells. The best characterized of these are the TH1 and TH2 subsets, however, other subsets include TH 17, Tregs (regulatory T cells), TFH (follicular TH cells) ${ }^{[10]}$.

\section{B-cell response}

The ability of B cells response to antigen depends on the Bcell antigen receptor. Activation of $B$ cells requires two signals. Depending on the nature of the antigen, B-cell activation proceeds by two different routes, one T-cell dependent (TH cells), the other T-cell independent activation ${ }^{[12]}$ (Figure.4).

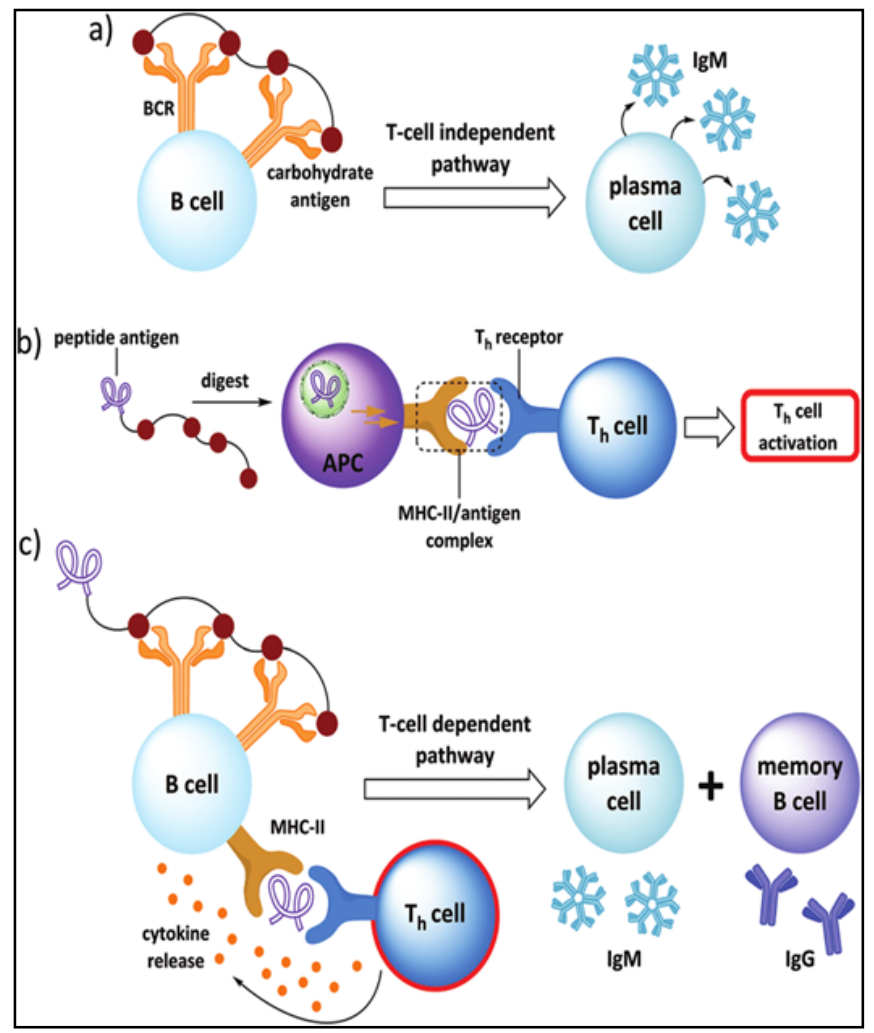

Fig 4: B-cell activation a) T-cell independent activation leading to production of plasma cells. b) and c) T-cell dependent pathway

leading to production of memory cells as well as activation of T-cells which results in enhanced immune response against pathogens

\section{T Cell-Independent Activation of B cells}

The BCR binds with high affinity, capable of responding to antigens even in absence of $T$ cells.

But these B cells donot mature and they are restricted to enter the memory pathway which is essential for production of antibodies. Thus it will not result in a secondary response to subsequent exposures to antigens.

\section{T Cell-Dependent Activation of B cells}

$\mathrm{T}$ cell-dependent activation of $\mathrm{B}$ cells is more complex, resulting in stronger immune response with the production of memory cells.

B-cells bind soluble antigens and part of antigen is now presented to T cells which provides activation signal to B-cell. The activators of T-cell include Gp39 and Gp34 which interact with the B-cell receptors CD40 ${ }^{[12]}$.

The coordination between $\mathrm{B}$ cells and helper $\mathrm{T}$ cells that are specific to the same antigen is referred to as linked recognition. Once activated by linked recognition, Tcells produce cytokines responsible for activation of $\mathrm{B}$ cell resulting in proliferation and differentiation of B-cells into plasma cells. Plasma cells are antibody factories that secrete large quantities of antibodies. After differentiation, the surface BCRs disappear and the plasma cell secretes pentameric IgM molecules that have the same antigen specificity as the BCRs. 


\section{Immunological Memory}

The most significant feature of an adaptive immune response is the establishment of a state of immunological memory. The ability of the immune system to respond to pathogens that have been encountered previously, and reflects the preexistence of a clonally expanded population of antigenspecific lymphocytes in a most rapid and effective manner. Memory responses, which are called secondary, tertiary, and so on, depending on the number of exposures to antigen, also differ qualitatively from primary responses.

The characteristics of antibodies produced in secondary and subsequent responses are distinct from those produced in the primary response to the same antigen. The concept was first recorded by the ancient Greeks and has been exploited routinely in vaccination programs for over 200 years

Immunological memory is mediated by conventional adaptive cells: B cells, $\mathrm{T}$ cells, that have differentiated to become "memory" populations.

Memory T cells generate a considerable number of effector T cells, capable of cytokine secretion and/or cytolytic activity, within hours of antigenic restimulation, whereas naïve $\mathrm{T}$ cells generate a smaller number of effectors and at a much slower pace.

Memory B cells are defined as long-lived and quiescent cells that are poised to quickly respond to antigen upon recall ${ }^{[14}$ 15]

\section{Conclusion}

The inflammatory events that develop in response to the bacterial challenge are protective by intent, but they result in the majority of tissue damage and breakdown that lead to the clinical signs of periodontitis. $\mathrm{T}$ and $\mathrm{B}$ cell subset, as well as their cytokine expression, plays a major role in the pathogenesis of the periodontal disease. The significance of adaptive immunity lies in the generation of memory cells or the ability of the system to record its experiences of encounters with various pathogens in a manner leading to even more effective and rapid responses with subsequent challenges with the same or similar infectious disease. For this reason, it is important to understand the different molecular and cellular mechanisms of the pathogenesis of periodontal disease, for making an opportune diagnosis, appropriate treatment and prognosis.

\section{References}

1. Darveau RP. Periodontitis: a polymicrobial disruption of host homeostasis. Nature Reviews Microbiology 2010;8(7):481-90.

2. Marshall JS, Warrington R, Watson W, Kim HL. An introduction to immunology and immunopathology. Allergy, Asthma \& Clinical Immunology 2018;14(2):1-0.

3. Medzhitov R, Janeway Jr CA. Innate immunity: impact on the adaptive immune response. Current opinion in immunology 1997;9(1):4-9.

4. Ahmed A, Saha B, Patwardhan A, Shivprasad S, Nandi D. The major players in adaptive immunity. Resonance 2009;14(5):455-71.

5. Teng YT. The role of acquired immunity and periodontal disease progression. Critical Reviews in Oral Biology \& Medicine 2003;14(4):237-52.

6. Deshpande N, Dave D. Antigen-presenting cells in periodontal disease. Indian Journal of Oral Sciences 2012;3(2):69-73.

7. Vyas JM, Van der Veen AG, Ploegh HL. The known unknowns of antigen processing and presentation. Nature
Reviews Immunology 2008;8(8):607-18.

8. Balaji S, Cholan K, John P, Victor D, Appukuttan D, Thangarasu V. Development and Activation of $\mathrm{T}$ cell subsets-An Overview from a Periodontal Perspective. European Journal of Molecular \& Clinical Medicine 2021;7(1):4489-501.

9. Bierer BE, Hahn WC. T cell adhesion, avidity regulation and signaling: a molecular analysis of CD2. InSeminars in immunology 1993;5(4):249-261. Academic Press.

10. Smith-Garvin JE, Koretzky GA, Jordan MS. T cell activation. Annual review of immunology 2009;27:591619.

11. Hwang JR, Byeon Y, Kim D, Park SG. Recent insights of $\mathrm{T}$ cell receptor-mediated signaling pathways for $\mathrm{T}$ cell activation and development. Experimental \& molecular medicine 2020;52(5):750-61.

12. Bonilla FA, Oettgen HC. Adaptive immunity. Journal of Allergy and Clinical Immunology 2010;125(2):S33-40.

13. Tew J, Engel D, Mangan D. Polyclonal B-cell activation in periodontitis. Journal of Periodontal Research 1989;24(4):225-41.

14. Crotty S, Ahmed R. Immunological memory in humans. In Seminars in immunology. Academic Press 2004;16(3):197-203.

15. Kurosaki T, Kometani K, Ise W. Memory B cells. Nature Reviews Immunology 2015;15(3):149-5. 